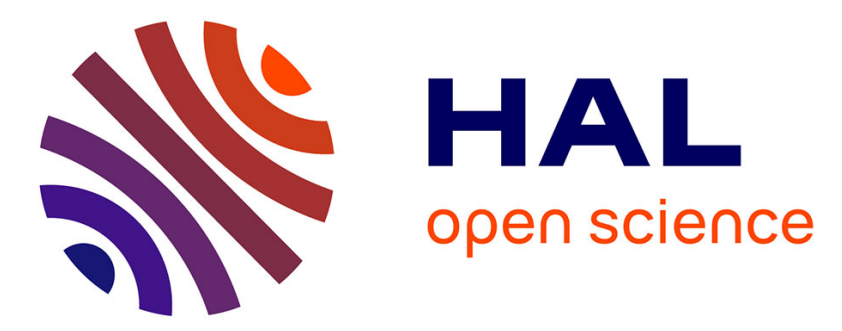

\title{
Sur la faible identité nationale des Italiens
}

Mario Caciagli, Emmanuel Négrier

\section{To cite this version:}

Mario Caciagli, Emmanuel Négrier. Sur la faible identité nationale des Italiens. Pôle Sud - Revue de science politique de l'Europe méridionale, 2001, État ou nation(s) ?, 14, pp.29-39. 10.3406/pole.2001.1103 . hal-02551176

\section{HAL Id: hal-02551176 https://hal.science/hal-02551176}

Submitted on 22 Apr 2020

HAL is a multi-disciplinary open access archive for the deposit and dissemination of scientific research documents, whether they are published or not. The documents may come from teaching and research institutions in France or abroad, or from public or private research centers.
L'archive ouverte pluridisciplinaire HAL, est destinée au dépôt et à la diffusion de documents scientifiques de niveau recherche, publiés ou non, émanant des établissements d'enseignement et de recherche français ou étrangers, des laboratoires publics ou privés.

\section{(이) $\$$}

Distributed under a Creative Commons Attribution - NonCommercial - NoDerivatives| 4.0 


\section{Sur la faible identité nationale des Italiens}

\section{Mr Mario Caciagli, Mr Emmanuel Négrier}

\section{Résumé}

During the last decade, there has been in Italy an important debate about the weekness of the Italian national belief. It was the consequence of the defiance of the Lega Nord towards the Italian State, as well as the break down of the First Republic. A lot of historical and political scientists have scrutinized the nature and the origines of such a problem: the backward unification, the exteriority of the State among the people, economical and social disparities as well as the survival of regional dialects, mentalities and political behaviors. However, investigations and sample surveys assess that such a weekness of national identity is not so intense. Moreover, they show a gap between State and Nation among italian people. Finally, this low sense of belonging to a Nation-State could be considered as a positive perspective, in the framework of the European integration process.

\section{Citer ce document / Cite this document :}

Caciagli Mario, Négrier Emmanuel. Sur la faible identité nationale des Italiens. In: Pôle Sud, n¹4, 2001. État ou nation(s) ? pp. 29-39;

doi : https://doi.org/10.3406/pole.2001.1103

https://www.persee.fr/doc/pole_1262-1676_2001_num_14_1_1103

Fichier pdf généré le 23/04/2018 


\title{
Sur la faible indentité nationale des Italiens
}

\author{
Mario Caciagli \\ Université de Florence
}

(Traduit de l'italien par Emmanuel Négrier)

\section{Résumé/Abstract}

Le défi que pose la Lega Nord à l'unité de l'État et l'effondrement de la première République ont ouvert en Italie un grand débat sur la faiblesse de la conscience nationale. L'intervention des médias s'est accompagnée d'ouvrages d'historiens et de politistes qui examinent la nature et les raisons du problème: l'unification tardive, l'extranéité de l'État pour les masses populaires, les fractures économiques et sociales, la survivance de dialectes et de mentalités régionales et la force des subcultures politiques. Les enquêtes et sondages affirment cependant que le déficit d'identité nationale, chez les Italiens, n'est pas aussi intense que ce qu'en attestent les intellectuels. Ce dont témoignent les Italiens, c'est d'un désajustement entre Etat et Nation. Mais le faible sens de l'appartenance à un État-nation n'ouvre-t-il pas une perspective favorable, dans le cadre du processus d'intégration européenne?

During the last decade, there has been in Italy an important debate about the weekness of the Italian national belief. It was the consequence of the defiance of the Lega Nord towards the Italian State, as well as the break down of the First Republic. A lot of historical and political scientists have scrutinized the nature and the origines of such a problem: the backward unification, the exteriority of the State among the people, economical and social disparities as well as the survival of regional dialects, mentalities and political behaviors. However, investigations and sample surveys assess that such a weekness of national identity is not so intense. Moreover, they show a gap between State and Nation among italian people. Finally, this low sense of belonging to a Nation-State could be considered as a positive perspective, in the framework of the European integration process.

\section{$\approx$ \\ Mots-Clés/Keywords}

État italien, histoire politique italienne, Lega Nord, Nation italienne, sentiment européen, subcultures

European consciousness, Italian Nation, Italian political history, Italian State, Lega Nord, subcultures 


\section{Le tricolore}

Le tricolore, blanc, rouge et vert, est comme on le sait le drapeau italien. Dans tous les Etats-nation contemporains, le drapeau est le symbole de l'identité nationale. Ainsi, le tricolore est, ou devrait être un des "lieux de mémoire" (Oliva, 1997) qui fondent l'identité nationale des Italiens. Or, si le tricolore semble, au moins à travers ce que nous transmet la documentation historique, avoir été très présent dans la scénographie publique de l'Italie monarchique et fasciste, il n'a pas connu une telle fortune dans la République démocratique. Nous verrons plus loin pourquoi.

Aujourd'hui cependant, le tricolore, désormais accompagné du drapeau bleu de l'Europe, est au fronton de tous les édifices publics italiens: ministères, préfectures, hôtels de région, de provinces et de communes, sièges d'organismes publics divers, sans oublier les écoles et universités. Ce furent les ministres de l'Instruction publique et de l'Intérieur du gouvernement Prodi, de centregauche et donc moins suspect de sentiment nationaliste, qui ordonnèrent que tous ces édifices d'État dussent arborer la bannière italienne. Nous verrons également plus loin pourquoi.
Une fois seulement tous les quatre ans les drapeaux italiens se déploient en nombre sur les places et avenues d'Italie, pendent des fenêtres et aux portes des cafés, quand l'Italia aimée, entendue comme l'équipe de football, joue et l'emporte dans les compétitions du championnat du monde de ce sport si populaire. C'est en cette seule occasion que des millions de tricolori inondent les cités et villages du bel paese, uniquement quand "onze frères en culotte et maillots azur", comme le disait le grand journaliste sportif Gianni Brera, se battent sur la pelouse d'un stade de foot, que les Italiens se reconnaissent en un destin commun.

\section{"Les Italiens sont les autres"}

En dehors de ces moments unitaires de ferveur sportive, les Italiens ne s'aiment pas généralement entre eux. Au contraire, ils sont le plus souvent prompts à critiquer sévèrement... les autres Italiens. Chacun d'entre eux, en fait, sait reconnaître les défauts et comportements négatifs de ceux au milieu desquels il lui revient de vivre, les Italiens précisément. Mais ceux auxquels on attribue de 
tels défauts et attitudes inconvenantes sont toujours les autres. "Les Italiens sont les autres" comme l'indique le titre du récent ouvrage d'un auteur connu, qui y a rassemblé des articles déjà publiés dans plusieurs grands quotidiens (Vassalli, 1998). Chaque italien, en tant qu'individu, estime se comporter correctement, tandis que les Italiens, si mal éduqués, si indisciplinés, si peu respectueux de la loi sont... les autres.

Aux Italiens il semble en somme manquer le sens d'une appartenance à une communauté et partant le sentiment d'une responsabilité collective.

Les Italiens ont d'eux-mêmes, en tant que peuple, une piètre estime. Elle ne pouvait qu'empirer dans les années 1990 sous l'effet de Tangentopoli, c'est-à-dire la découverte de la grande corruption qui érodait le système politique et le tissu social même de la République. Le simple citoyen s'est naturellement senti innocent: les coupables étaient les autres, en l'occurrence une classe politique entière.

Avec Tangentopoli la crise de la Première République est devenue majeure. L'effondrement des grands partis historiques, les coups portés au cœur de l'État par la criminalité organisée et, par-dessus tout, l'essor au Nord d'un fort mouvement d'abord autonomiste puis sécessionniste, ont mis au premier rang de l'ordre du jour les rapports des Italiens avec leur État et leur nation.

C'est le défi présenté par la Lega Nord qui a réveillé la problématique de la "nation italienne". Si "Nation" est un ensemble de valeurs et d'attitudes communes et partagées qui créent une identité collective, beaucoup se sont pris à se demander si tout cela n'était pas très faible dans la conscience italienne.

De là, la décision du gouvernement italien d'inviter les offices publics à arborer le tricolore à leurs façades se voulait être une première réponse au problème.

\section{Le lamento des intellectuels}

La naissance et l'expansion du phénomène léguiste, accompagnant donc le crépuscule de la première république, ont ouvert un grand débat parmi les intellectuels sur la cause de la faiblesse de la conscience nationale des Italiens et des remèdes à y apporter.

Déjà, en 1990, un journaliste célèbre, Giorgio Bocca, préoccupé par les premiers et surprenants succès des mouvements "localistes", avait publié un livre intitulé L'Italie désunie. Selon Bocca, qui a repris ce thème dans les années ultérieures, cette désunion est due à la fracture entre un Nord productif et un Sud parasite. C'est surtout cette fracture qui conduirait au fait que l'Italie soit une nation sans un peuple et une culture homogènes (Bocca, 1990). Si nous cessons d'être une Nation, titre d'un livre paru en 1993, exprime pour la première fois le désarroi et les préoccupations de bien des intellectuels. L'auteur en était Gian Enrico Rusconi, un universitaire qui, ces années-là, avait consacré plusieurs interventions à ce thème. La Lega Nord représente pour Rusconi une menace gravissime pour une communauté qui, par la faute de ses intellectuels et de ses dirigeants, ne sait pas conjuguer "Démocratie" et "Nation". Se réclamant du "patriotisme constitutionnel" de Jürgen Habermas, Rusconi s'attend à ce que l'Italie finisse par être ou devenir "une Nation de citoyens" (Rusconi, 1993).

A quoi sert l'Italie?, se demandait au contraire la partie monographique d'un fascicule de 1994 d'une revue de géopolitique, Limes, qui dans son éditorial rappelait que "nous les Italiens sommes habitués à l'idée de ne pas être une Nation" (Limes, 1994, p. 7). 
Dans son livre, la même année, Italia addio? Unité et désunion de 1860 à nos jours, l'historien Aurelio Lepre a reconstitué les causes de l'intense et diffus rejet de l'Unité nationale et a rappelé que le problème était déjà évident aux intellectuels italiens de la deuxième moitié du siècle dernier, alors qu'il fut simplement déplacé chez les générations suivantes (Lepre, 1994).

Voilà quels furent les ouvrages principaux qui ont ouvert un débat passionné, qui dure encore aujourd'hui. Ne pouvant ici le restituer entièrement et convoquer toutes les voix qui y ont pris part, rappelons simplement les opérations éditoriales qui, au cours de la seule année 1998, lui ont donné une grande vigueur.

Les Italiens sans Italie est le titre, par exemple, de l'ouvrage d'un historien de l'antiquité, Aldo Schiavone, qui a recherché les lointaines origines du désarroi italien (Schiavone, 1998). Un philosophe, Remo Bodei, a réuni quelques-uns de ses travaux sur le sujet sous le titre Le nous divisé (Bodei, 1998). Une maison d'édition réputée, spécialisée en science sociale, Il Mulino de Bologne, a créé précisément une collection de poche appelée "Lidentité italienne" afin, comme l'indique le catalogue, de raconter "de quelle façon les Italiens sont devenus ce qu'ils sont aujourd'hui... pour comprendre l'origine, les contenus et le sens de leur identité individuelle et collective". Le premier volume de la série s'intitule L'Identité italienne. L'auteur, Ernesto Galli della Loggia, y analyse la scission entre "identité nationale et identité italienne" (p. 66) et déplore lui aussi "le faible sentiment que les Italiens ont d'être une nation et les rares circonstances à l'occasion desquelles ils démontrent l'être vraiment (p. 157) (Galli della Loggia, 1998).

Si ceux-ci sont seulement les livres les plus intéressants qui déplorent le faible sentiment national italien, le public transalpin a été littéralement inondé par une vague d'articles de quotidiens, d'éditoriaux de revues scientifiques, de débats télévisés et radiophoniques. Il n'est pas exclu, en outre, que derrière les bannières tricolores des façades scolaires et universitaires, bien des enseignants aient développé plus d'une fois l'argument lors des leçons, ou sollicité les élèves à y réfléchir à l'occasion des cours d'histoire et d'italien.

En somme, nous avons assisté et assistons encore à un chœur ou les cris émus l'ont disputé à des réflexions plus approfondies.

\section{Un peu d'histoire}

L'explication principale de la faiblesse de l'identité des Italiens est, depuis plus d'un siècle, toujours la même: le retard d'unification du pays. Après des siècles de domination étrangère et de division en plusieurs Etats de dimensions diverses, et, surtout, de différents degrés de développement économique et social, on sait que c'est seulement en 1861, avec la naissance du Regno d'Italia, que se réalisa l'unité nationale. Le Regno d'Italia naquit par l'initiative d'un Etat aux confins, le Regno di Sardegna, dont le barycentre était les Alpes et la capitale Turin, la plus italienne des villes françaises, selon une définition ostensiblement ironique.

Le premier roi d'Italie parlait fort mal l'italien et beaucoup de ses hauts fonctionnaires se retrouvèrent à administrer un pays qu'ils estimaient étranger. La création du nouveau système politico-institutionnel fut l'œuvre d'une oligarchie restreinte, qui peina à former une bourgeoisie moderne en mesure d'incarner une identité nationale. Ce fut l'un des protagonistes de l'unification, Massimo D'Azeglio, qui prononça la phrase que tous les écoliers sont encore 
contraints d'apprendre: "l'Italie est faite, il faut maintenant faire les Italiens".

Les paysans, c'est-à-dire la grande majorité de la population, demeurèrent en marge du processus d'unification, soit à cause des conditions misérables dans lesquelles ils se trouvaient, en particulier dans le Sud, soit à cause de leur total manque de droit politique. L'Église catholique, indignée par la perte de Rome, monta ces paysans contre l'État unitaire, libéral et laïc, avec de notables succès, notamment dans le NordEst. La majorité des paysans découvrit l'Italie seulement lorsqu'elle devint de la chair à canons, au cours de la première guerre mondiale.

L'Italie continua longtemps à ressembler à un manteau d'Arlequin. La survivance de dialectes, de coutumes et de mentalités différentes et contradictoires divisa pour des décennies les Italiens. D'autre part, la langue italienne était parlée par peu de gens. L'école publique et la conscription militaire se révéleront insuffisantes pour former une identité nationale.

Les Italiens restèrent également divisés en termes de forme de développement (ou de non développement) économique, et d'émergence de cultures politiques divergentes. Parmi d'autres, trois grandes fractures divisèrent l'État libéral, contribuant ainsi à sa crise définitive après la première guerre mondiale.

La plus profonde des trois fractures était de caractère territorial, entre le Nord et le Sud, le premier lancé sur la voie de l'industrialisation tandis que le second restait pauvre et arriéré. Cette fracture est encore aujourd'hui ouverte dans le corps de l'Etatnation, et contribue à rendre difficile une entente entre tous les Italiens. Si ce type de fracture n'est pas absolument spécifique à l'Italie, elle a eu, par sa profondeur et sa durée, un poids énorme dans les relations entre Italiens.

Elle existe encore aujourd'hui sous l'appellation "la question méridionale", dont les origines remontent donc à la constitution de l'État italien lui-même. Les régions de l'ancien règne des Deux-Siciles n'étaient pas seulement, au moment de l'unification, bien plus pauvres et arriérées que celles du Nord, mais leurs habitants étaient aussi largement plus influencés par des valeurs traditionnelles. Dans un ouvrage qui a suscité un grand débat en Italie, un politiste américain a fait remonter aux communes du Moyen-Âge la culture civique des Italiens du Nord et au féodalisme des barons méridionaux le faible sens de l'État et de la nation des Italiens du Sud (Putnam, 1993).

De vastes secteurs de la société méridionale considérèrent l'avènement du régime italo-piémontais comme une occupation étrangère. Dans toutes les strates sociales, et spécialement dans la grande masse des paysans et du sous-prolétariat urbain, se forma une défiance à l'égard de l'État qui se traduisit souvent en véritable adversité. Les citoyens du Mezzogiorno, au lieu de s'intégrer au sein de la nouvelle entité collective - c'est-à-dire l'État-nation dénommé Regno d'Italia -, renforcèrent leurs prédispositions culturelles : familiarisme et parentèle, individualisme et particularisme.

Les deux autres fractures qui marquèrent la société italienne à la fin du XIXe siècle se retrouvent plus fréquemment dans l'histoire des autres pays européens : la fracture sociale, entre capitalistes et travailleurs, et le clivage confessionnel, entre catholiques et non-catholiques. Mais ces deux fractures, là aussi, ont pris en Italie un tour dramatique, en devenant un obstacle à la formation d'une identité nationale. La classe ouvrière ne s'intégra pas à l'État ou à la Nation, et 
construisit sa propre contre-culture; les catholiques, sous l'influence du clergé et d'un Vatican hostiles au nouvel État - au moins jusqu'aux premières décennies du $\mathrm{XX}^{e}$ siècle - se refusèrent à prendre part à la vie politique et furent donc à l'origine d'une autre contre-culture. Pendant le Regno d'Italia, en lieu et place du drapeau tricolore, les bannières rouges du prolétariat et blanches du mouvement catholique furent les symboles politiques dans lesquels beaucoup d'Italiens se reconnurent.

Le fascisme, dictature inspirée d'une idéologie nationaliste, s'escrima à intégrer de force les Italiens dans l'Etat-nation qu'il avait conquis. Les mythes et rites du régime cherchèrent à leur inculquer le sens d'une appartenance à une nation unique. Avec la création de héros nationaux, avec l'éducation scolastique ou le sport (les équipes sportives qui représentaient l'Italie, à commencer par celle de football, furent qualifiées de "nationales" par décision du régime), le fascisme visait à consolider cette si faible identité italienne, mais finit par sombrer dans le nationalisme.

Les conséquences du nationalisme fasciste, la guerre et la défaite, provoquèrent une réaction de rejet de la part des Italiens quant à l'idée même de Nation. Non seulement leur identité nationale était notablement affaiblie dans l'après-guerre, mais l'énorme majorité des Italiens se convainquit que le patriotisme et le sentiment national était une idée dangereuse qui n'engendrait que conflits et tragédies.

La démocratie républicaine, au travers de la domination des trois grands partis de masse - la Démocratie chrétienne, les partis socialiste et communiste, qui n'avaient certes pas Nation et Nationalité parmi leurs valeurs fondatrices - fut loin de s'appuyer sur de telles ressources. Elles furent au contraire considérées comme risquées, à l'heure où les grands partis avaient pour projet l'intégration des masses au sein du nouveau système politique.

\section{Les cultures politiques sub- nationales de la Première République}

Au cours du long après-guerre italien, les termes "Nation" et "Patrie" furent non seulement bannis du vocabulaire politique, mais encore du discours commun, à la seule exception de l'extrême-droite.

Les deux grands partis dominant le système politique y contribuèrent notablement. Pour des raisons différentes et parfois opposées, ni la Démocratie chrétienne ni le Parti communiste ne pouvaient être sensibles à la consolidation d'une identité nationale. Le parti catholique, dont on sait que les ancêtres n'avaient voulu reconnaître l'État-nation des libéraux, se référait plutôt au Vatican ou aux Etats-Unis. Le Parti communiste avait comme référence l'Union soviétique, la "patrie du socialisme": le parti avait accolé le tricolore à la bannière rouge sur son effigie, mais l'internationalisme et l'expectative de la révolution prolétarienne mortifiaient l'identité nationale.

Les deux grandes forces politiques ont continué de s'appuyer sur les deux subcultures politiques antérieures au fascisme. L'aspect le plus important de cette continuité est que les deux subcultures se renforcèrent, durant la Première République, dans leur composante territoriale. La subculture rouge, tout comme la blanche, sont devenues de plus en plus territoriales, contribuant ainsi davantage a l'affirmation d'identités territoriales qu'à une identité nationale.

Toutes les analyses du système politique italien, et spécialement celles dédiées au com- 
portement électoral et aux organisations partisanes, ont constamment appliqué les catégories de "régions rouges" (la majorité des provinces d'Emilie-Romagne, de Toscane, d'Ombrie) et de "régions blanches" (les provinces voisines des Alpes et, en particulier, presque toute la Vénétie). La culture politique des citoyens de ces régions s'est alors parée des valeurs, symboles et modèles de comportement que leur conférait une identité forte et distincte. Mais cette identité était de fait idéologico-territoriale, nonnationale; par définition, elle s'appuyait sur des "cultures partielles", contradictoires avec une culture nationale. Ces régions, les rouges jusqu'à il y a peu, ont eu un rapport très faible, voire de conflit ouvert à l'égard du centre, et donc de l'État et de la Nation.

Dans la République s'est également perpétuée l'autre grande contradiction au sein de l'Etat-nation italien: la fracture NordSud. Même en perspective historique, après un siècle et demi d'État unitaire, il n'est pas possible de dire quelle est la cause et quels sont les effets attribuables soit à la fracture socio-économique (Sud arriéré contre Nord développé), soit à la fracture culturelle (des valeurs et modèles de comportement divers, voire contradictoires, y compris en termes politiques).

La rareté des ressources économiques et les déséquilibres sociaux, d'un côté, l'individualisme et le particularisme de l'autre, ont continué à conditionner les mentalités et l'action des citoyens du Sud, au sein même de la République démocratique. Ces caractères ont trouvé une forme politique définitive dans le clientélisme, en tant que mode d'organisation du consensus et de l'exercice du pouvoir.

Dans les cultures subnationales du Nord se sont développées des formes de conscience collective et des modalités de vie communautaire, notamment par l'activité même des grands partis. Tous deux, de diverses manières, ont contribué à intégrer les masses du Nord à l'État, et donc à les rapprocher de l'idée d'État-nation, même si l'œuvre reste inachevée.

Au Sud, l'État reste encore perçu comme lointain et hostile. Il n'y est reconnu que comme dispensateur de faveurs - postes, subsides et subventions. Un seul parti y est né, et c'était le parti de gouvernement, la Démocratie chrétienne. Mais c'était un parti singulier, le parti clientélaire de masse, qui distribuait les ressources sur la base de critères particularistes. Chez les citoyens du Sud, le sens de l'appartenance à une communauté s'est développé sur des bases matérielles et non comme l'acceptation de valeurs partagées.

Pour tout cela, les Italiens du Nord se sont sentis différents de ceux du Sud et vice-versa; pour tout cela il a été difficile de consolider le sens d'une appartenance commune. De cette grave fracture est né le phénomène de la Lega Nord. La Lega exhorte les septentrionaux à combattre contre l'État centraliste, parce qu'elle est hostile à la réalité politique et sociale et la culture du Mezzogiorno. Selon les Italiens du Nord qui se reconnaissent dans la Lega, l'État transfere trop de richesses créés au Nord vers un Sud parasite, sous la forme de transferts et de subventions. L'explosion de la criminalité organisée, qui a ses bases au Sud et qui contrôle plusieurs zones méridionales comme un "État dans l'État", a justifié peutêtre encore davantage l'attitude de la Lega, et a en tout cas creusé le fossé entre Nord et Sud. "Nous sommes deux nations" est devenu, pour des raisons différentes, le cri de bien des Italiens. La Lega Nord exige, sur cette base, la sécession de l'Italie d'un nouvel État-nation: la "Padanie" (Biorcio, 1997).

Enfin, dans le cadre persistant de fragmentation dans un difficile processus de 
construction stato-nationale en Italie, il ne faut naturellement pas oublier les minorités ethniques. En Italie, on parle allemand dans le Sud-Tyrol, français dans le Val d'Aoste. Ces populations vivent dans des régions qui bénéficient de statuts spéciaux qui leur garantissent la préservation d'une langue, de coutumes et d'une auto-organisation politique (cf. les partis Südtiroler Volkspartei et Union Valdôtaine). En Sardaigne, qui jouit aussi d'un statut autonome et a un parti autonomiste (le Partito Sardo d'Azione), l'usage officiel du sarde, langue romane ayant sa propre dignité, est également garanti.

Ces minorités et institutions de la périphérie contribuent à leur manière à fragmenter les sentiments d'appartenance collective des Italiens.

\section{Lieux communs et confron- tations empiriques}

Cette sommaire reconstitution historique du système italien semble donc nous conduire à douter de l'existence d'une solide identité nationale chez les Italiens. La tradition historique, les cultures politiques, les déséquilibres territoriaux et la faiblesse des institutions justifient un tel constat. Le système "Italia" est-il alors menacé par des antagonismes irréductibles et, directement, par une prochaine sécession?

En dépit de tout ce que nous avons considéré opportun de rappeler, nous estimons que la situation italienne est moins dramatique que ce que les opinions des élites conduisent à penser. Sur la division de l'Italie et des Italiens, les craintes des intellectuels et politiques sont probablement exagérées. L'accusation faite à la majorité de leurs compatriotes de "se sentir peu Italiens" n'est pas très fondée.

Nous avons déjà indiqué que les premiers intéressés, les Italiens, ne semblaient que peu perturbés par le grand débat des années 1990. Même les scandales de la Première République et sa crise ne paraissent avoir provoqué que des réactions temporaires de rejet quant à l'appartenance à la nation italienne.

La lecture des intellectuels, en outre, semble reprendre beaucoup de lieux communs qui amplifient les différences et les comportements des Italiens d'une manière caricaturale, afin d'en faire de réels stéréotypes (Sciolla, 1997). La lecture en termes de "familiarisme" et de "particularisme" de la conscience collective italienne reflète un banal sens commun qui s'exprime en proverbes et en maximes (et en images cinématographiques). Trop souvent enfin l'on recourt au "caractère national" qui est, comme on le sait, une catégorie scientifiquement très confuse et politiquement bien dangereuse.

S'il est vrai que les Italiens ont peu d'estime d'eux-mêmes, cela ne signifie pas nécessairement qu'ils manquent d'identité nationale. Les raisons historiques et sociales de leur distance à l'égard d'un sentiment national commun sont certes évidentes et il nous a semblé opportun de les rappeler. Mais les jugements ordinaires n'ont, souvent, pas de confirmation empirique.

Le terrain le plus solide de l'analyse empirique, en fait, ne paraît pas fournir les indices les plus négatifs sur l'identité nationale des Italiens. Il résulte des sondages des éléments qui, pour n'être pas toujours d'une interprétation limpide et certaine, permettent de tirer des leçons intéressantes. Voyons lesquelles.

Un sondage conduit en septembre 1994 par l'agence SWG pour la revue Limes semblait démentir le fait que les sentiments localistes et régionalistes aient affaibli le sens de l'appartenance nationale. Au contraire, l'orgueil national" apparaissait comme l'orientation la plus partagée par les Italiens, sans que cela contredise d'ailleurs 
une forte identification avec une région ou une ville en particulier. Les interprétations de ce sondage conduisaient ainsi à "une identité composite et articulée", en mesure de faire coexister des référentiels divers (Diamanti \& Segatti, 1994).

Un autre chercheur qui, au cours d'une étude conduite sur douze années, avait progressivement enregistré une faiblesse croissante de l'identité nationale chez les Italiens, a découvert une nette inversion de tendance à partir de 1994. Après cette date, les Italiens démontraient que leur sentiment d'appartenance à la communauté nationale s'était renforcé. Peut-être considéraient-ils que la période négative de l'histoire de leur pays, marquée par la corruption et la fin de la Première République, était désormais derrière eux; ou bien réagissaient-ils ainsi à la provocation centrifuge de la Lega Nord. Il résultait également de cette étude que les identités régionales, pour être fortes, et cela plus au Nord qu'au Sud, n'en étaient pas moins d'une intensité inférieure à l'identité nationale (Barbé, 1997).

Un autre sondage de 1997 confirmait que $80 \%$ des interviewés se sentaient "fiers d'être Italiens", un pourcentage désormais supérieur à celui enregistré quelques années auparavant. Plus, parmi ceux qui envisageaient la sécession du Nord du reste de l'Italie, une majorité se déclarait toutefois fière d'être italien (Segatti, 1999).

Enfin, une consultation conduite en mai 1998 par l'Institut Poster de Vicenza pour le compte du quotidien Il Sole 24 ore a donné des résultats qui se prêtent à des interprétations pour le moins non-univoques. Ainsi, le fait que $57 \%$ des interviewés aient déclaré leur disponibilité à "faire de grands sacrifices pour défendre leur pays" semblait, pour le directeur de l'Institut Poster "confirmer le déficit d'identité nationale des Italiens" (Dia- manti, 1998). Mais $57 \%$ doivent-ils être considérés comme un chiffre faible ou au contraire élevé, après le grand et préoccupant débat des dernières années? Et les $28 \%$ qui ne sont pas disposés à "faire de grands sacrifices": est-ce peu ou beaucoup? Quel serait le pourcentage atteint pas les autres pays?

Un autre commentateur de ce même sondage souligne que les données démontrent qu'"une identité nationale apparaît plausible là où existe une communauté locale de référence" (Marini, 1998). Eh bien cela non plus ne nous paraît pas devoir être accueilli négativement.

\section{Une perspective post-nationale}

Ainsi l'homme de la rue, le citoyen des sondages ne dirait pas la vérité dans ses réactions? Les intellectuels savent-ils réellement tout et mieux? La contradiction entre le sentiment d'appartenance nationale des citoyens de base et l'analyse des intellectuels cacherait-elle un paradoxe?

Le fait est que la fierté d'être italien est due, pour l'homme de la rue, à la beauté du paysage péninsulaire, à la richesse de son patrimoine artistique et à son art de vivre (cuisine comprise). L'orgueil se fonde donc sur des éléments culturels que les Italiens évaluent positivement et qui leur permettent de s'y reconnaître en tant que Nation.

Ce qu'il manque aux Italiens est la capacité ou la possibilité, compte tenu de l'expérience historique, d'identifier l'État avec la Nation. Ce qui est demeuré toujours incertain dans l'histoire et donc dans les consciences est précisément la perception d'une construction "statonationale".

À bien y regarder, c'est cela que déplorent les intellectuels qui connaissent leur métier et l'histoire de leur pays mieux que les citoyens 
sondés. Leur lamento est donc justifié s'il concerne le désajustement entre État et Nation, la faible diffusion auprès des Italiens d'une culture civique acceptée et partagée. On doit alors dire que les Italiens n'ont pas tant une faible identité nationale, mais plutôt un faible sens de l'État.

C'est donc l'idée que la symbiose entre État et Nation serait nécessaire qui fonde les jugements négatifs sur la condition des Italiens. Mais cette idée (ou peut-être cette idéologie) n'appartient-elle pas désormais au passé de l'aventure européenne? L'Étatnation n'est il pas une construction en crise, même ailleurs?

S'il en est ainsi, il est possible de conclure comme suit. Si l'identité nationale des Italiens, mesurée à partir de leur identification avec leur État, est faible, l'a toujours été, et l'est devenue plus encore avec la crise de la Première République, ceci ne peut être considéré comme un drame. Au contraire, la question d'un faible sentiment d'appartenance à un État-nation ouvre, à la fin du vingtième siècle, des perspectives tout sauf négatives.

Nous retenons personnellement que la multiplicité des cultures politiques italiennes pourrait produire, comme cela est arrivé par le passé, une forme d'intégration solide. Dans la complexe société contemporaine, une différenciation consciente et acceptée peut être en réalité la meilleure voie pour une identité collective.

Même certains des intellectuels qui se sont confrontés à cette problématique trouvent aujourd'hui des aspects positifs à cette division des Italiens. Ainsi Bodei, dont j'ai déjà parlé à propos de son "moi divisé", reconnaît que "les divisions et les mémoires historiques fragmentées peuvent même représenter une ressource, un ressort qui favorise la dynamique interne de la société italienne" (Bodei, 1998).
Cette ressource apparaît encore plus évidente si on la rapporte au futur proche des Etats européens. Qui est convaincu de la crise définitive de la forme Etat-nation qui a dominé l'Europe des quatre derniers siècles (mais en Italie, comme en Allemagne, il s'est réalisé il y a à peine plus d'un siècle), ne peut qu'accepter non seulement comme inévitable, mais souhaitable l'articulation interne de structures unitaires désormais dépassées. Schiavone, un autre des protagonistes du grand débat, a donc raison. Voici sa proposition: "Ne plus chercher à construire une Nation adaptée à nos temps, mais simplement, pour ainsi dire, l'outrepasser... et porter notre pays entier au niveau d'une autre dimension de développement. C'est l'idée européenne qui nous contraint à un voyage mental au-delà de l'État-nation" (Schiavone, 1998, pp. 135-136).

En somme, face à la perspective d'une Union européenne toujours plus intégrée et d'une "Europe des Régions" qui a déjà une consistance économique et quasi-institutionnelle, la différenciation articulée de l'Italie et des Italiens, qui produit une aussi faible identité nationale, peut être une ressource importante pour la constitution d'une identité collective qui demeure en-deçà ou qui va au-delà de l'État-nation.

Les sondages que nous avons mentionnés plus haut indiquent, enfin, que les Italiens regardent avec intérêt l'émergence d'une identité européenne. Toutes les données de l'Eurobaromètre, à leur tour, signalent que les Italiens sont les plus "européistes" de tous les Européens, dépassant les Allemands après leur unification. Les Italiens semblent en somme avoir commencé à développer une identité post-nationale pour laquelle la faiblesse du sentiment national s'avère être une force, si elle s'inscrit dans une si peu méprisable perspective. 


\section{Références}

Barbé C., "Il drastico irrobustimento dell'identità nazionale in Italia. Un'analisi empirica comparata", Quaderni di sociologia, $\mathrm{n}^{\circ} 13,1997$.

Biorcio R., La Padania promessa. La storia, le idee e la logica d'azione della Lega Nord, Milan, Il Saggiatore, 1997.

Bocca G., La disunita d'Italia, Milan, Garzanti, 1990.

Bodei R., Il noi diviso. Ethos e idee dell'Italia repubblicana, Turin, Einaudi, 1998.

Diamanti I., "Un difficile slalom tra disincanto e impegno sociale", Il Sole/24 ore, 8 luglio 1998.

Diamanti I., Segatti P., "Orgogliosi di essere italiani”, Limes, n 4, 1994.

Galli della Loggia E., L'identità italiana, Bologne, Il Mulino, 1998.

Lepre A., Italia addio? Unità e disunità dal 1860 a oggi, Milan, Mondadori, 1994.

Limes, "A che serve l'Italia. Perché siamo una Nazione", numéro monographique, n 4, 1994.

Marini D., "Il patriottismo "divide" l'Italia", Il Sole/24 ore, 8 luglio 1998.

Oliva G., "Il tricolore", in Isnenghi M., (dir.), I luoghi della memoria. Simboli e miti dell'Italia unita, RomeBari, Laterza, 1996.

Putnam R., Making Democracy Work, Princeton, Princeton University Press, 1993.

Rusconi G. E., Se cessiamo di essere una Nazione, Bologne, Il Mulino, 1993.

Schiavone A., Italiani senza Italia, Turin, Einaudi, 1998.

Sciolla L., Italiani. Stereotipi di casa nostra, Bologne, Il Mulino, 1997.

Segatti P., "Perché è debole la coscienza nazionale degli italiani”, Il Mulino, n 1, 1999.

Vassalli S., Gli italiani sono gli altri, Milan, Baldini \& Castoldi, 1998. 